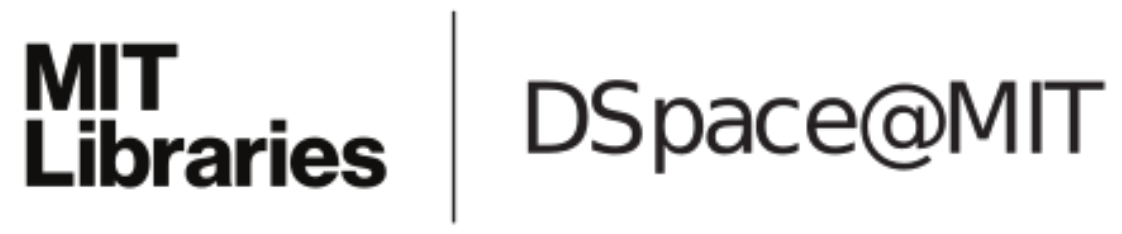

\author{
MIT Open Access Articles
}

Automatic rigging and animation of $3 D$ characters

The MIT Faculty has made this article openly available. Please share how this access benefits you. Your story matters.

Citation: Ilya Baran and Jovan Popovic. 2007. Automatic rigging and animation of 3D characters. ACM Trans. Graph. 26, 3, Article 72 (July 2007).

As Published: http://dx.doi.org/10.1145/1276377.1276467

Publisher: Association for Computing Machinery (ACM)

Persistent URL: http://hdl.handle.net/1721.1/100396

Version: Author's final manuscript: final author's manuscript post peer review, without publisher's formatting or copy editing

Terms of Use: Article is made available in accordance with the publisher's policy and may be subject to US copyright law. Please refer to the publisher's site for terms of use. 


\title{
Automatic Rigging and Animation of 3D Characters
}

\author{
Ilya Baran* Jovan Popović ${ }^{\dagger}$ \\ Computer Science and Artificial Intelligence Laboratory \\ Massachusetts Institute of Technology
}

\begin{abstract}
Animating an articulated 3D character currently requires manual rigging to specify its internal skeletal structure and to define how the input motion deforms its surface. We present a method for animating characters automatically. Given a static character mesh and a generic skeleton, our method adapts the skeleton to the character and attaches it to the surface, allowing skeletal motion data to animate the character. Because a single skeleton can be used with a wide range of characters, our method, in conjunction with a library of motions for a few skeletons, enables a user-friendly animation system for novices and children. Our prototype implementation, called Pinocchio, typically takes under a minute to rig a character on a modern midrange PC.
\end{abstract}

CR Categories: I.3.7 [Computer Graphics]: Three-Dimensional Graphics and Realism-Animation

Keywords: Animation, Deformations, Geometric Modeling

\section{Introduction}

Modeling in 3D is becoming much easier than before. User-friendly systems such as Teddy [Igarashi et al. 1999] and Cosmic Blobs (http://www.cosmicblobs.com/) have made the creation of 3D characters accessible to novices and children. Bringing these static shapes to life, however, is still not easy. In a conventional skeletal animation package, the user must rig the character manually. This requires placing the skeleton joints inside the character and specifying which parts of the surface are attached to which bone. The tedium of this process makes simple character animation more difficult than it could be.

We envision a system that eliminates this tedium to make animation more accessible for children, educators, researchers, and other non-expert animators. For example, a child should be able to model a unicorn, click the "Quadruped Gallop" button, and watch the unicorn start galloping. To support this functionality, we need a method (as shown in Figure 1) that takes a character, a skeleton, and a motion of that skeleton as input, and outputs the moving character. The missing portion is the rigging: motion transfer has been addressed in prior work [Gleicher 2001].

Our algorithm consists of two main steps: skeleton embedding and skin attachment. Skeleton embedding computes the joint positions of the skeleton inside the character by minimizing a penalty

*e-mail: ibaran@mit.edu

†e-mail: jovan@csail.mit.edu

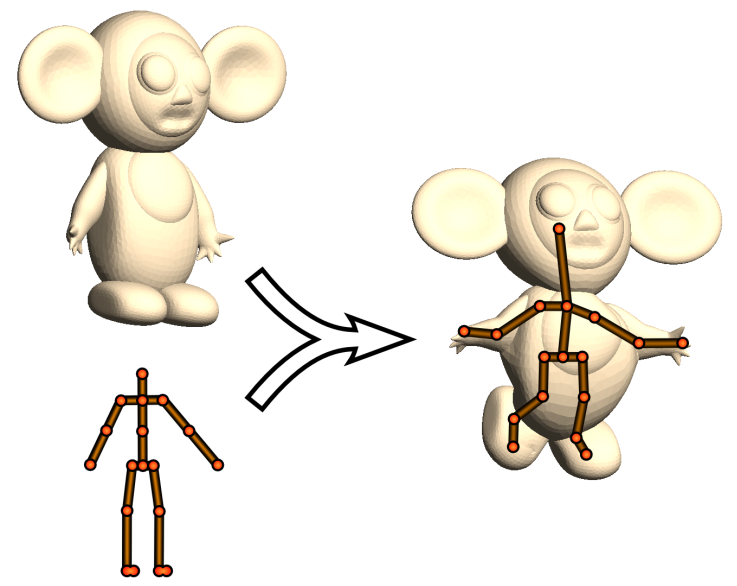

Figure 1: The automatic rigging method presented in this paper allowed us to implement an easy-to-use animation system, which we called Pinocchio. In this example, the triangle mesh of a jolly cartoon character is brought to life by embedding a skeleton inside it and applying a walking motion to the initially static shape.

function. To make the optimization problem computationally feasible, we first embed the skeleton into a discretization of the character's interior and then refine this embedding using continuous optimization. The skin attachment is computed by assigning bone weights based on the proximity of the embedded bones smoothed by a diffusion equilibrium equation over the character's surface.

Our design decisions relied on three criteria, which we also used to evaluate our system:

- Generality: A single skeleton is applicable to a wide variety of characters: for example, our method can use a generic biped skeleton to rig an anatomically correct human model, an anthropomorphic robot, and even something that has very little resemblance to a human.

- Quality: The resulting animation quality is comparable to that of modern video games.

- Performance: The automatic rigging usually takes under one minute on an everyday PC.

A key design challenge is constructing a penalty function that penalizes undesirable embeddings and generalizes well to new characters. For this, we designed a maximum-margin supervised learning method to combine a set of hand-constructed penalty functions. To ensure an honest evaluation and avoid overfitting, we tested our algorithm on 16 characters that we did not see or use during development. Our algorithm computed a good rig for all but 3 of these characters. For each of the remaining cases, one joint placement hint corrected the problem.

We simplify the problem by making the following assumptions. The character mesh must be the boundary of a connected volume. 
The character must be given in approximately the same orientation and pose as the skeleton. Lastly, the character must be proportioned roughly like the given skeleton.

We introduce several new techniques to solve the automatic rigging problem:

- A maximum-margin method for learning the weights of a linear combination of penalty functions based on examples, as an alternative to hand-tuning (Section 3.3).

- An $A^{*}$-like heuristic to accelerate the search for an optimal skeleton embedding over an exponential search space (Section 3.4).

- Use of Laplace's diffusion equation to generate weights for attaching mesh vertices to the skeleton using linear blend skinning (Section 4). This method could also be useful in existing $3 \mathrm{D}$ packages.

Our prototype system, called Pinocchio, rigs the given character using our algorithm. It then transfers a motion to the character using online motion retargetting [Choi and Ko 2000] to eliminate footskate by constraining the feet trajectories of the character to the feet trajectories of the given motion.

\section{Related Work}

Character Animation Most prior research in character animation, especially in 3D, has focused on professional animators; very little work is targeted at novice users. Recent exceptions include Motion Doodles [Thorne et al. 2004] as well as the work of Igarashi et al. on spatial keyframing [2005b] and as-rigid-as-possible shape manipulation [2005a]. These approaches focus on simplifying animation control, rather than simplifying the definition of the articulation of the character. In particular, a spatial keyframing system expects an articulated character as input, and as-rigid-as-possible shape manipulation, besides being $2 \mathrm{D}$, relies on the constraints to provide articulation information. The Motion Doodles system has the ability to infer the articulation of a 2D character, but their approach relies on very strong assumptions about how the character is presented.

Skeleton Extraction Although most skeleton-based prior work on automatic rigging focused on skeleton extraction, for our problem, we advocate skeleton embedding. A few approaches to the skeleton extraction problem are representative. Teichmann and Teller [1998] extract a skeleton by simplifying the Voronoi skeleton with a small amount of user assistance. Liu et al. [2003] use repulsive force fields to find a skeleton. In their paper, Katz and Tal [2003] describe a surface partitioning algorithm and suggest skeleton extraction as an application. The technique in Wade [2000] is most similar to our own: like us, they approximate the medial surface by finding discontinuities in the distance field, but they use it to construct a skeleton tree.

For the purpose of automatically animating a character, however, skeleton embedding is much more suitable than extraction. For example, the user may have motion data for a quadruped skeleton, but for a complicated quadruped character, the extracted skeleton is likely to have a different topology. The anatomically appropriate skeleton generation by Wade [2000] ameliorates this problem by techniques such as identifying appendages and fitting appendage templates, but the overall topology of the resulting skeleton may still vary. For example, for the character in Figure 1, ears may be mistaken for arms. Another advantage of embedding over extraction is that the given skeleton provides information about the expected structure of the character, which may be difficult to obtain from just the geometry. So although we could use an existing skeleton extraction algorithm and embed our skeleton into the extracted one, the results would likely be undesirable. For example, the legs of the character in Figure 1 would be too short if a skeleton extraction algorithm were used.

Template Fitting Animating user-provided data by fitting a template has been successful in cases when the model is fairly similar to the template. Most of the work has been focused on human models, making use of human anatomy specifics, e.g. [Moccozet et al. 2004]. For segmenting and animating simple 3D models of characters and inanimate objects, Anderson et al. [2000] fit voxel-based volumetric templates to the data.

Skinning Almost any system for mesh deformation (whether surface based [Lipman et al. 2005; Yu et al. 2004] or volume based [Zhou et al. 2005]) can be adapted for skeleton-based deformation. Teichmann and Teller [1998] propose a spring-based method. Unfortunately, at present, these methods are unsuitable for real-time animation of even moderate size meshes. Because of its simplicity and efficiency (and simple GPU implementation), and despite its quality shortcomings, linear blend skinning (LBS), also known as skeleton subspace deformation, remains the most popular method used in practice.

Most real-time skinning work, e.g. [Kry et al. 2002; Wang et al. 2007], has focused on improving on LBS by inferring the character articulation from multiple example meshes. However, such techniques are unsuitable for our problem because we only have a single mesh. Instead, we must infer articulation by using the given skeleton as an encoding of the likely modes of deformation, not just as an animation control structure.

To our knowledge, the problem of finding bone weights for LBS from a single mesh and a skeleton has not been sufficiently addressed in the literature. Previous methods are either mesh resolution dependent [Katz and Tal 2003] or the weights do not vary smoothly along the surface [Wade 2000], causing artifacts on highresolution meshes. Some commercial packages use proprietary methods to assign default weights. For example, Autodesk Maya 7 assigns weights based solely on the vertex proximity to the bone, ignoring the mesh structure, which results in serious artifacts when the mesh intersects the Voronoi diagram faces between logically distant bones.

\section{Skeleton Embedding}

Skeleton embedding resizes and positions the given skeleton to fit inside the character. This can be formulated as an optimization problem: "compute the joint positions such that the resulting skeleton fits inside the character as nicely as possible and looks like the given skeleton as much as possible." For a skeleton with $s$ joints (by "joints," we mean vertices of the skeleton tree, including leaves), this is a $3 s$-dimensional problem with a complicated objective function. Solving such a problem directly using continuous optimization is infeasible.

Pinocchio therefore discretizes the problem by constructing a graph whose vertices represent potential joint positions and whose edges are potential bone segments. This is challenging because the graph must have few vertices and edges, and yet capture all potential bone paths within the character. The graph is constructed by packing spheres centered on the approximate medial surface into the character and by connecting sphere centers with graph edges. Pinocchio then finds the optimal embedding of the skeleton into this graph with respect to a discrete penalty function. It uses the discrete solution as a starting point for continuous optimization.

To help with optimization, the given skeleton can have a little extra information in the form of joint attributes: for example, joints that should be approximately symmetric should be marked as such; also some joints can be marked as "feet," indicating that they should be placed near the bottom of the character. We describe the attributes Pinocchio uses in a supplemental document[Baran and 


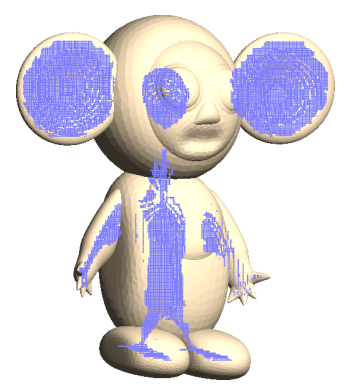

Figure 2: Approximate Medial Surface

Popović 2007a]. These attributes are specific to the skeleton but are independent of the character shape and do not reduce the generality of the skeletons.

\subsection{Discretization}

Before any other computation, Pinocchio rescales the character to fit inside an axis-aligned unit cube. As a result, all of the tolerances are relative to the size of the character.

Distance Field To approximate the medial surface and to facilitate other computations, Pinocchio computes a trilinearly interpolated adaptively sampled signed distance field on an octree [Frisken et al. 2000]. It constructs a kd-tree to evaluate the exact signed distance to the surface from an arbitrary point. It then constructs the distance field from the top down, starting with a single octree cell and splitting a cell until the exact distance is within a tolerance $\tau$ of the interpolated distance. We found that $\tau=0.003$ provides a good compromise between accuracy and efficiency for our purposes. Because only negative distances (i.e. from points inside the character) are important, Pinocchio does not split cells that are guaranteed not to intersect the character's interior.

Approximate Medial Surface Pinocchio uses the adaptive distance field to compute a sample of points approximately on the medial surface (Figure 2). The medial surface is the set of $C^{1}$ discontinuities of the distance field. Within a single cell of our octree, the interpolated distance field is guaranteed to be $C^{1}$, so it is necessary to look at only the cell boundaries. Pinocchio therefore traverses the octree and for each cell, looks at a grid (of spacing $\tau$ ) of points on each face of the cell. It then computes the gradient vectors for the cells adjacent to each grid point-if the angle between two of them is $120^{\circ}$ or greater, it adds the point to the medial surface sample. We impose the $120^{\circ}$ condition because we do not want the "noisy" parts of the medial surface-we want the points where skeleton joints are likely to lie. For the same reason, Pinocchio filters out the sampled points that are too close to the character surface (within $2 \tau$ ). Wade discusses a similar condition in Chapter 4 of his thesis [2000].

Sphere Packing To pick out the graph vertices from the medial surface, Pinocchio packs spheres into the character as follows: it sorts the medial surface points by their distance to the surface (those that are farthest from the surface are first). Then it processes these points in order and if a point is outside all previously added spheres, adds the sphere centered at that point whose radius is the distance to the surface. In other words, the largest spheres are added first, and no sphere contains the center of another sphere (Figure 3). Although the procedure described above takes $O(n b)$ time in the worst case (where $n$ is the number of points, and $b$ is the final number of spheres inserted), worst case behavior is rarely seen because most points are processed while there is a small number of large

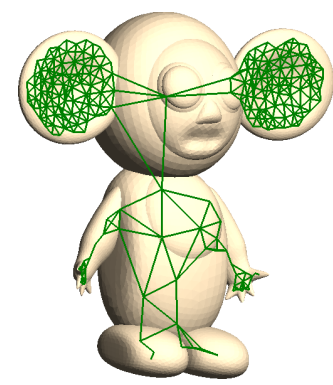

Figure 4: Constructed Graph

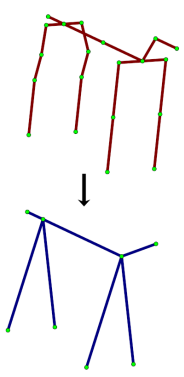

Figure 5: The original and reduced quadruped skeleton spheres. In fact, this step typically takes less than $1 \%$ of the time of the entire algorithm.

Graph Construction The final discretization step constructs the edges of the graph by connecting some pairs of sphere centers (Figure 4). Pinocchio adds an edge between two sphere centers if the spheres intersect. We would also like to add edges between spheres that do not intersect if that edge is well inside the surface and if that edge is "essential." For example, the neck and left shoulder spheres of the character in Figure 3 are disjoint, but there should still be an edge between them. The precise condition Pinocchio uses is that the distance from any point of the edge to the surface must be at least half of the radius of the smaller sphere, and the closest sphere centers to the midpoint of the edge must be the edge endpoints. The latter condition is equivalent to the requirement that additional edges must be in the Gabriel graph of the sphere centers (see e.g. [Jaromczyk and Toussaint 1992]). While other conditions can be formulated, we found that the Gabriel graph provides a good balance between sparsity and connectedness.

Pinocchio precomputes the shortest paths between all pairs of vertices in this graph to speed up penalty function evaluation.

\subsection{Reduced Skeleton}

The discretization stage constructs a geometric graph $G=(V, E)$ into which Pinocchio needs to embed the given skeleton in an optimal way. The skeleton is given as a rooted tree on $s$ joints. To reduce the degrees of freedom, for the discrete embedding, Pinocchio works with a reduced skeleton, in which all bone chains have been merged (all degree two joints, such as knees, eliminated), as shown in Figure 5. The reduced skeleton thus has only $r$ joints. This works because once Pinocchio knows where the endpoints of a bone chain are in $V$, it can compute the intermediate joints by taking the shortest path between the endpoints and splitting it in accordance with the proportions of the unreduced skeleton. For the humanoid skeleton we use, for example, $s=18$, but $r=7$; without a reduced skeleton, the optimization problem would typically be intractable.

Therefore, the discrete skeleton embedding problem is to find the embedding of the reduced skeleton into $G$, represented by an $r$ tuple $\mathbf{v}=\left(v_{1}, \ldots, v_{r}\right)$ of vertices in $V$, which minimizes a penalty function $f(\mathbf{v})$ that is designed to penalize differences in the embedded skeleton from the given skeleton.

\subsection{Discrete Penalty Function}

The discrete penalty function has great impact on the generality and quality of the results. A good embedding should have the proportions, bone orientations, and size similar to the given skeleton. The paths representing the bone chains should be disjoint, if possible. Joints of the skeleton may be marked as "feet," in which case they should be close to the bottom of the character. Designing a penalty function that satisfies all of these requirements simultaneously is 
difficult. Instead we found it easier to design penalties independently and then rely on learning a proper weighting for a global penalty that combines each term.

The Setup We represent the penalty function $f$ as a linear combination of $k$ "basis" penalty functions: $f(\mathbf{v})=\sum_{i=1}^{k} \gamma_{i} b_{i}(\mathbf{v})$. Pinocchio uses $k=9$ basis penalty functions constructed by hand. They penalize short bones, improper orientation between joints, length differences in bones marked symmetric, bone chains sharing vertices, feet away from the bottom, zero-length bone chains, improper orientation of bones, degree-one joints not embedded at extreme vertices, and joints far along bone-chains but close in the graph [Baran and Popović 2007a]. We determine the weights $\Gamma=\left(\gamma_{1}, \ldots, \gamma_{k}\right)$ semi-automatically via a new maximum margin approach inspired by support vector machines.

Suppose that for a single character, we have several example embeddings, each marked "good" or "bad". The basis penalty functions assign a feature vector $\mathbf{b}(\mathbf{v})=\left(b_{1}(\mathbf{v}), \ldots, b_{k}(\mathbf{v})\right)$ to each example embedding $\mathbf{v}$. Let $\mathbf{p}_{1}, \ldots, \mathbf{p}_{m}$ be the $k$-dimensional feature vectors of the good embeddings and let $\mathbf{q}_{1}, \ldots, \mathbf{q}_{n}$ be the feature vectors of the bad embeddings.

Maximum Margin To provide context for our approach, we review the relevant ideas from the theory of support vector machines. See Burges [1998] for a much more complete tutorial. If our goal were to automatically classify new embeddings into "good" and "bad" ones, we could use a support vector machine to learn a maximum margin linear classifier. In its simplest form, a support vector machine finds the hyperplane that separates the $\mathbf{p}_{i}$ 's from the $\mathbf{q}_{i}$ 's and is as far away from them as possible. More precisely, if $\Gamma$ is a $k$-dimensional vector with $\|\Gamma\|=1$, the classification margin of the best hyperplane normal to $\Gamma$ is $\frac{1}{2}\left(\min _{i=1}^{n} \Gamma^{T} \mathbf{q}_{i}-\max _{i=1}^{m} \Gamma^{T} \mathbf{p}_{i}\right)$. Recalling that the total penalty of an embedding $\mathbf{v}$ is $\Gamma^{T} \mathbf{b}(\mathbf{v})$, we can think of the maximum margin $\Gamma$ as the one that best distinguishes between the best "bad" embedding and the worst "good" embedding in the training set.

In our case, however, we do not need to classify embeddings, but rather find a $\Gamma$ such that the embedding with the lowest penalty $f(\mathbf{v})=\Gamma^{T} \mathbf{b}(\mathbf{v})$ is likely to be good. To this end, we want $\Gamma$ to distinguish between the best "bad" embedding and the best "good" embedding, as illustrated in Figure 6. We therefore wish to maximize the optimization margin (subject to $\|\Gamma\|=1$ ), which we define as:

$$
\min _{i=1}^{n} \Gamma^{T} \mathbf{q}_{i}-\min _{i=1}^{m} \Gamma^{T} \mathbf{p}_{i}
$$

Because we have different characters in our training set, and because the embedding quality is not necessarily comparable between different characters, we find the $\Gamma$ that maximizes the minimum margin over all of the characters.

Our approach is similar to margin-based linear structured classification [Taskar et al. 2003], the problem of learning a classifier that to each problem instance (cf. character) assigns the discrete label (cf. embedding) that minimizes the dot product of a weights vector with basis functions of the problem instance and label. The key difference is that structured classification requires an explicit loss function (in our case, the knowledge of the quality of all possible skeleton embeddings for each character in the training set), whereas our approach only makes use of the loss function on the training labels and allows for the possibility of multiple correct labels. This possibility of multiple correct skeleton embeddings prevented us from formulating our margin maximization problem as a convex optimization problem. However, multiple correct skeleton embeddings are necessary for our problem in cases such as the hand joint being embedded into different fingers.

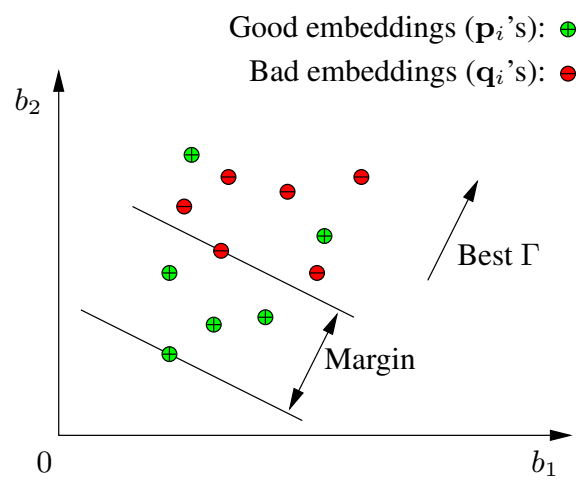

Figure 6: Illustration of optimization margin: marked skeleton embeddings in the space of their penalties $\left(b_{i}\right.$ 's)

Learning Procedure The problem of finding the optimal $\Gamma$ does not appear to be convex. However, an approximately optimal $\Gamma$ is acceptable, and the search space dimension is sufficiently low (9 in our case) that it is feasible to use a continuous optimization method. We use the Nelder-Mead method [Nelder and Mead 1965] starting from random $\Gamma$ 's. We start with a cube $[0,1]^{k}$, pick random normalized $\Gamma$ 's, and run Nelder-Mead from each of them. We then take the best $\Gamma$, use a slightly smaller cube around it, and repeat.

To create our training set of embeddings, we pick a training set of characters, manually choose $\Gamma$, and use it to construct skeleton embeddings of the characters. For every character with a bad embedding, we manually tweak $\Gamma$ until a good embedding is produced. We then find the maximum margin $\Gamma$ as described above and use this new $\Gamma$ to construct new skeleton embeddings. We manually classify the embeddings that we have not previously seen, augment our training set with them, and repeat the process. If $\Gamma$ eventually stops changing, as happened on our training set, we use the found $\Gamma$. It is also possible that a positive margin $\Gamma$ cannot be found, indicating that the chosen basis functions are probably inadequate for finding good embeddings for all characters in the training set.

For training, we used 62 different characters (Cosmic Blobs models, free models from the web, scanned models, and Teddy models), and $\Gamma$ was stable with about 400 embeddings. The weights we learned resulted in good embeddings for all of the characters in our training set; we could not accomplish this by manually tuning the weights. Examining the optimization results and the extremal embeddings also helped us design better basis penalty functions.

Although this process of finding the weights is labor-intensive, it only needs to be done once. According to our tests, if the basis functions are carefully chosen, the overall penalty function generalizes well to both new characters and new skeletons. Therefore, a novice user will be able to use the system, and more advanced users will be able to design new skeletons without having to learn new weights.

\subsection{Discrete Embedding}

Computing a discrete embedding that minimizes a general penalty function is intractable because there are exponentially many embeddings. However, if it is easy to estimate a good lower bound on $f$ from a partial embedding (of the first few joints), it is possible to use a branch-and-bound method. Pinocchio uses this idea: it maintains a priority queue of partial embeddings ordered by their lower bound estimates. At every step, it takes the best partial embedding from the queue, extends it in all possible ways with the next joint, and pushes the results back on the queue. The first full embedding extracted is guaranteed to be the optimal one. This is essentially the $A^{*}$ algorithm on the tree of possible embeddings. To speed up 
the process and conserve memory, if a partial embedding has a very high lower bound, it is rejected immediately and not inserted into the queue.

Although this algorithm is still worst-case exponential, it is fast on most real problems with the skeletons we tested. We considered adapting an approximate graph matching algorithm, like [Gold and Rangarajan 1996], which would work much faster and enable more complicated reduced skeletons. However, computing the exact optimum simplified penalty function design and debugging.

The joints of the skeleton are given in order, which induces an order on the joints of the reduced skeleton. Referring to the joints by their indices (starting with the root at index 1 ), we define the parent function $p_{R}$ on the reduced skeleton, such that $p_{R}(i)$ (for $1<i \leq r)$ is the index of the parent of joint $i$. We require that the order in which the joints are given respects the parent relationship, i.e. $p_{R}(i)<i$.

Our penalty function $(f)$ can be expressed as the sum of independent functions of bone chain endpoints $\left(f_{i}\right.$ 's $)$ and a term $\left(f_{D}\right)$ that incorporates the dependence between different joint positions. The dependence between joints that have not been embedded can be ignored to obtain a lower bound on $f$. More precisely, $f$ can be written as:

$$
f\left(v_{1}, \ldots, v_{r}\right)=\sum_{i=2}^{r} f_{i}\left(v_{i}, v_{p_{R}(i)}\right)+\sum_{i=2}^{r} f_{D}\left(v_{1}, \ldots, v_{i}\right) .
$$

A lower bound when the first $k$ joints are embedded is then:

$$
\begin{array}{r}
\sum_{i=2}^{k} f_{i}\left(v_{i}, v_{p_{R}(i)}\right)+\sum_{i=2}^{k} f_{D}\left(v_{1}, \ldots, v_{i}\right)+ \\
+\sum_{\left\{i>k \mid p_{R}(i) \leq k\right\}} \min _{v_{i} \in V} f_{i}\left(v_{i}, v_{p_{R}(i)}\right)
\end{array}
$$

If $f_{D}$ is small compared to the $f_{i}$ 's, as is often the case for us, the lower bound is close to the true value of $f$.

Because of this lower bound estimate, the order in which joints are embedded is very important to the performance of the optimization algorithm. High degree joints should be embedded first because they result in more terms in the rightmost sum of the lower bound, leading to a more accurate lower bound. For example, our biped skeleton has only two joints of degree greater than two, so after Pinocchio has embedded them, the lower bound estimate includes $f_{i}$ terms for all of the bone chains.

Because there is no perfect penalty function, discrete embedding will occasionally produce undesirable results (see Model 13 in Figure 9). In such cases it is possible for the user to provide manual hints in the form of constraints for reduced skeleton joints. For example, such a hint might be that the left hand of the skeleton should be embedded at a particular vertex in $G$ (or at one of several vertices). Embeddings that do not satisfy the constraints are simply not considered by the algorithm.

\subsection{Embedding Refinement}

Pinocchio takes the optimal embedding of the reduced skeleton found by discrete optimization and reinserts the degree-two joints by splitting the shortest paths in $G$ in proportion to the given skeleton. The resulting skeleton embedding should have the general shape we are looking for, but typically, it will not fit nicely inside the character. Also, smaller bones are likely to be incorrectly oriented because they were not important enough to influence the discrete optimization. Embedding refinement corrects these problems by minimizing a new continuous penalty function (Figure 7).

For the continuous optimization, we represent the embedding of the skeleton as an $s$-tuple of joint positions $\left(q_{1}, \ldots, q_{s}\right)$ in $\mathbb{R}^{3}$. Because we are dealing with an unreduced skeleton, and discrete optimization has already found the correct general shape, the penalty

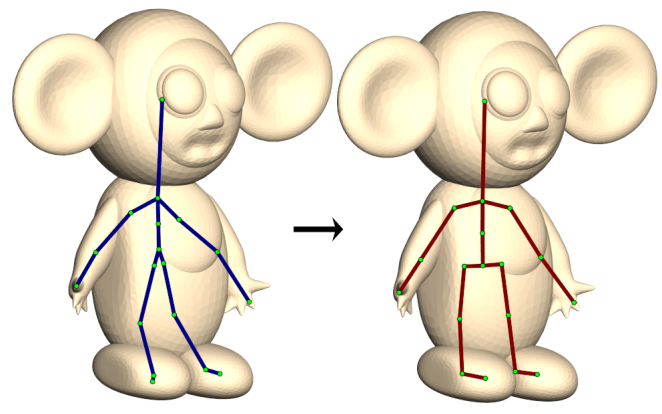

Figure 7: The embedded skeleton after discrete embedding (blue) and the results of embedding refinement (dark red)

function can be much simpler than the discrete penalty function. The continuous penalty function $g$ that Pinocchio tries to minimize is the sum of penalty functions over the bones plus an asymmetry penalty:

$$
g\left(q_{1}, \ldots, q_{s}\right)=\alpha_{A} g^{A}\left(q_{1}, \ldots, q_{s}\right)+\sum_{i=2}^{s} g_{i}\left(q_{i}, q_{p_{S}(i)}\right)
$$

where $p_{S}$ is the parent function for the unreduced skeleton (analogous to $p_{R}$ ). Each $g_{i}$ penalizes bones that do not fit inside the surface nicely, bones that are too short, and bones that are oriented differently from the given skeleton: $g_{i}=\alpha_{S} g_{i}^{S}+\alpha_{L} g_{i}^{L}+\alpha_{O} g_{i}^{O}$. Unlike the discrete case, we choose the $\alpha$ 's by hand because there are only four of them [Baran and Popović 2007a].

Any continuous optimization technique [Gill et al. 1989] should produce good results. Pinocchio uses a gradient descent method that takes advantage of the fact that there are relatively few interactions. As a subroutine, it uses a step-doubling line search: starting from a given point (in $\mathbb{R}^{3 s}$ ), it takes steps in the given optimization direction, doubling step length until the penalty function increases. Pinocchio intersperses a line search in the gradient direction with line searches in the gradient direction projected onto individual bones. Repeating the process 10 times is usually sufficient for convergence.

\section{Skin Attachment}

The character and the embedded skeleton are disconnected until skin attachment specifies how to apply deformations of the skeleton to the character mesh. Although we could make use of one of the various mesh editing techniques for the actual mesh deformation, we choose to focus on the standard linear blend skinning (LBS) method because of its widespread use. If $\mathbf{v}_{j}$ is the position of vertex $j, \mathbf{T}^{i}$ is the transformation of the $i^{\text {th }}$ bone, and $w_{j}^{i}$ is the weight of the $i^{\text {th }}$ bone for vertex $j$, LBS gives the position of the transformed vertex $j$ as $\sum_{i} w_{j}^{i} \mathbf{T}^{i}\left(\mathbf{v}_{j}\right)$. The attachment problem is finding bone weights $\mathbf{w}^{i}$ for the vertices - how much each bone transform affects each vertex.

There are several properties we desire of the weights. First of all, they should not depend on the mesh resolution. Second, for the results to look good, the weights need to vary smoothly along the surface. Finally, to avoid folding artifacts, the width of a transition between two bones meeting at a joint should be roughly proportional to the distance from the joint to the surface. Although a scheme that assigns bone weights purely based on proximity to bones can be made to satisfy these properties, such schemes will often fail because they ignore the character's geometry: for example, part of the torso may become attached to an arm. Instead, we use the analogy to heat equilibrium to find the weights. Suppose we 


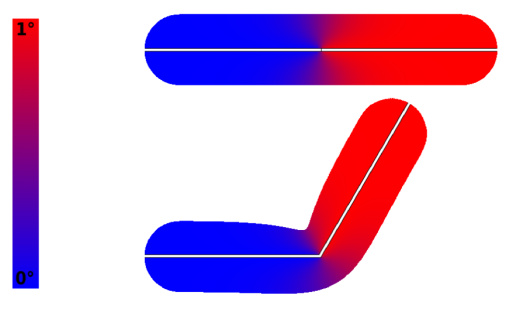

Figure 8: Top: heat equilibrium for two bones. Bottom: the result of rotating the right bone with the heat-based attachment

treat the character volume as an insulated heat-conducting body and force the temperature of bone $i$ to be 1 while keeping the temperature of all of the other bones at 0 . Then we can take the equilibrium temperature at each vertex on the surface as the weight of bone $i$ at that vertex. Figure 8 illustrates this in two dimensions.

Solving for heat equilibrium over a volume would require tessellating the volume and would be slow. Therefore, for simplicity, Pinocchio solves for equilibrium over the surface only, but at some vertices, it adds the heat transferred from the nearest bone. The equilibrium over the surface for bone $i$ is given by $\frac{\partial \mathbf{w}^{i}}{\partial t}=$ $\Delta \mathbf{w}^{i}+\mathbf{H}\left(\mathbf{p}^{i}-\mathbf{w}^{i}\right)=0$, which can be written as

$$
-\Delta \mathbf{w}^{i}+\mathbf{H} \mathbf{w}^{i}=\mathbf{H} \mathbf{p}^{i},
$$

where $\Delta$ is the discrete surface Laplacian, calculated with the cotangent formula [Meyer et al. 2003], $\mathbf{p}^{i}$ is a vector with $p_{j}^{i}=1$ if the nearest bone to vertex $j$ is $i$ and $p_{j}^{i}=0$ otherwise, and $\mathbf{H}$ is the diagonal matrix with $H_{j j}$ being the heat contribution weight of the nearest bone to vertex $j$. Because $\Delta$ has units of length ${ }^{-2}$, so must $\mathbf{H}$. Letting $d(j)$ be the distance from vertex $j$ to the nearest bone, Pinocchio uses $H_{j j}=c / d(j)^{2}$ if the shortest line segment from the vertex to the bone is contained in the character volume and $H_{j j}=0$ if it is not. It uses the precomputed distance field to determine whether a line segment is entirely contained in the character volume. For $c \approx 0.22$, this method gives weights with similar transitions to those computed by finding the equilibrium over the volume. Pinocchio uses $c=1$ (corresponding to anisotropic heat diffusion) because the results look more natural. When $k$ bones are equidistant from vertex $j$, heat contributions from all of them are used: $p_{j}$ is $1 / k$ for all of them, and $H_{j j}=k c / d(j)^{2}$.

Equation (1) is a sparse linear system, and the left hand side matrix $-\Delta+\mathbf{H}$ does not depend on $i$, the bone we are interested in. Thus we can factor the system once and back-substitute to find the weights for each bone. Botsch et al. [2005] show how to use a sparse Cholesky solver to compute the factorization for this kind of system. Pinocchio uses the TAUCS [Toledo 2003] library for this computation. Note also that the weights $\mathbf{w}^{i}$ sum to 1 for each vertex: if we sum (1) over $i$, we get $(-\Delta+\mathbf{H}) \sum_{i} \mathbf{w}^{i}=\mathbf{H} \cdot \mathbf{1}$, which yields $\sum_{i} \mathbf{w}^{i}=\mathbf{1}$.

It is possible to speed up this method slightly by finding vertices that are unambiguously attached to a single bone and forcing their weight to 1 . An earlier variant of our algorithm did this, but the improvement was negligible, and this introduced occasional artifacts.

\section{Results}

We evaluate Pinocchio with respect to the three criteria stated in the introduction: generality, quality, and performance. To ensure an objective evaluation, we use inputs that were not used during development. To this end, once the development was complete, we tested Pinocchio on 16 biped Cosmic Blobs models that we had not previously tried.
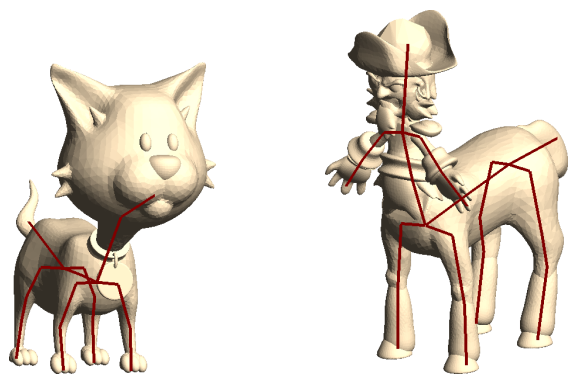

Figure 10: A centaur pirate with a centaur skeleton embedded looks at a cat with a quadruped skeleton embedded
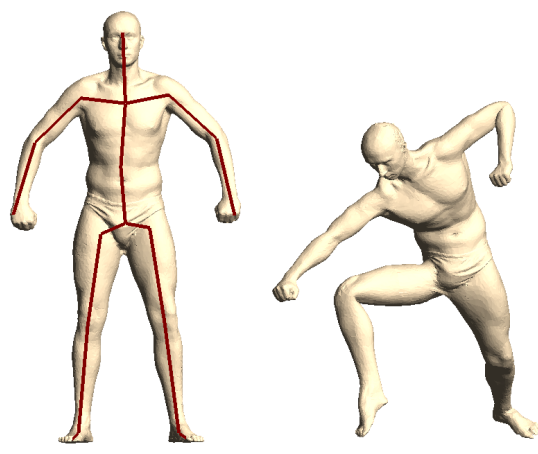

Figure 11: The human scan on the left is rigged by Pinocchio and is posed on the right by changing joint angles in the embedded skeleton. The well-known deficiencies of LBS can be seen in the right knee and hip areas.

\subsection{Generality}

Figure 9 shows our 16 test characters and the skeletons Pinocchio embedded. The skeleton was correctly embedded into 13 of these models ( $81 \%$ success). For Models 7, 10 and 13, a hint for a single joint was sufficient to produce a good embedding.

These tests demonstrate the range of proportions that our method can tolerate: we have a well-proportioned human (Models 1-4, 8), large arms and tiny legs (6; in 10, this causes problems), and large legs and small arms (15; in 13, the small arms cause problems). For other characters we tested, skeletons were almost always correctly embedded into well-proportioned characters whose pose matched the given skeleton. Pinocchio was even able to transfer a biped walk onto a human hand, a cat on its hind legs, and a donut.

The most common issues we ran into on other characters were:

- The thinnest limb into which we may hope to embed a bone has a radius of $2 \tau$. Characters with extremely thin limbs often fail because the the graph we extract is disconnected. Reducing $\tau$, however, hurts performance.

- Degree 2 joints such as knees and elbows are often positioned incorrectly within a limb. We do not know of a reliable way to identify the right locations for them: on some characters they are thicker than the rest of the limb, and on others they are thinner.

Although most of our tests were done with the biped skeleton, we have also used other skeletons for other characters (Figure 10).

\subsection{Quality}

Figure 11 shows the results of manually posing a human scan using our attachment. Our video [Baran and Popović 2007b] demonstrates the quality of the animation produced by Pinocchio. 


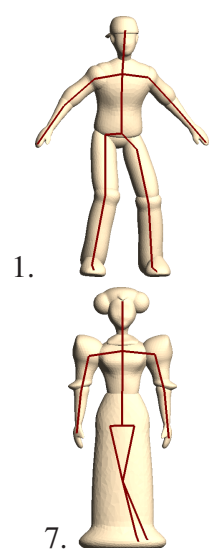

8.

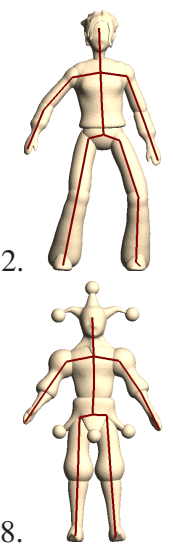

13.

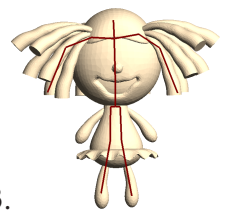

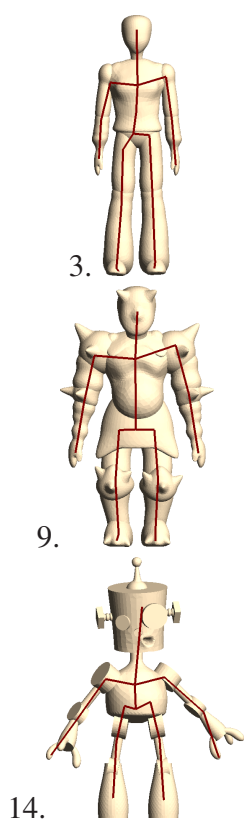
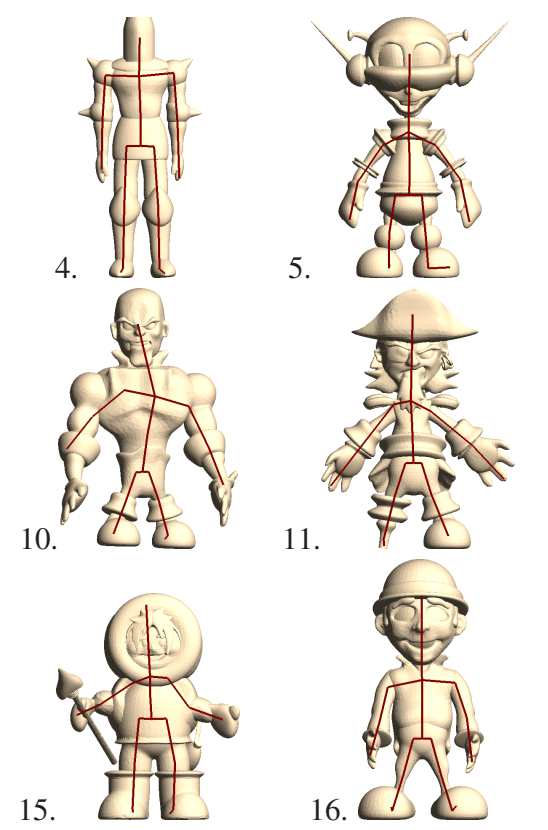

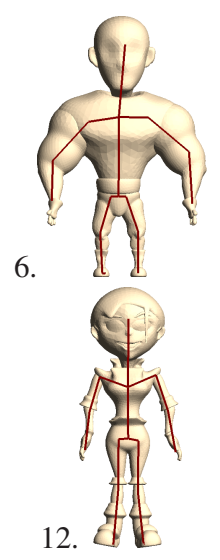

Figure 9: Test Results for Skeleton Embedding

\begin{tabular}{|r|c|c|c|c|}
\hline Model & 3 & 10 & 11 & Mean \\
\hline Number of Vertices & 19,001 & 34,339 & 56,856 & 33,224 \\
\hline Discretization Time & $10.3 \mathrm{~s}$ & $25.8 \mathrm{~s}$ & $68.2 \mathrm{~s}$ & $24.3 \mathrm{~s}$ \\
Embedding Time & $1.4 \mathrm{~s}$ & $29.1 \mathrm{~s}$ & $5.7 \mathrm{~s}$ & $5.2 \mathrm{~s}$ \\
Attachment Time & $0.9 \mathrm{~s}$ & $1.9 \mathrm{~s}$ & $3.2 \mathrm{~s}$ & $1.8 \mathrm{~s}$ \\
\hline Total Time & $12.6 \mathrm{~s}$ & $56.8 \mathrm{~s}$ & $77.1 \mathrm{~s}$ & $31.3 \mathrm{~s}$ \\
\hline
\end{tabular}

Table 1: Timings for three representative models and the mean over our 16 character test set

The quality problems of our attachment are a combination of the deficiencies of our automated weights generation as well as those inherent in LBS. A common class of problems is caused by Pinocchio being oblivious to the material out of which the character is made: the animation of both a dress and a knight's armor has an unrealistic, rubbery quality. Other problems occur at difficult areas, such as hips and the shoulder/neck region, where hand-tuned weights could be made superior to those found by our algorithm.

\subsection{Performance}

Table 1 shows the fastest and slowest timings of Pinocchio rigging the 16 models discussed in Section 5.1 on a $1.73 \mathrm{MHz}$ Intel Core Duo with 1GB of RAM. Pinocchio is single-threaded so only one core was used. We did not run timing tests on denser models because someone wishing to create real-time animation is likely to keep the triangle count low. Also, because of our volume-based approach, once the distance field has been computed, subsequent discretization and embedding steps do not depend on the given mesh size.

For the majority of models, the running time is dominated by the discretization stage, and that is dominated by computing the distance field. Embedding refinement takes about 1.2 seconds for all of these models, and the discrete optimization consumes the rest of the embedding time.

\section{Conclusion and Future Work}

We have presented the first method for automatically rigging an unfamiliar character for skeletal animation. In conjunction with ex- isting techniques, it allows a user to go from a static mesh to an animated character quickly and effortlessly. We have shown that using this method, Pinocchio can animate a wide range of characters. We also believe that some of our techniques, such as finding LBS weights and using examples to learn the weights of a linear combination of penalty functions, can be useful in other contexts.

We have several ideas for improving Pinocchio that we have not yet tried. Discretization could be improved by packing ellipsoids instead of spheres. Although this is more difficult, we believe it would greatly reduce the size of the graph, resulting in faster and higher quality discrete embeddings. Animation quality can be improved with a better skinning model [Kavan and Žára 2005] (although possibly at the cost of performance). One approach would be to use a technique [Wang et al. 2007] that corrects LBS errors by using example meshes, which we could synthesize using slower, but more accurate deformation techniques. A more involved approach would be automatically building a tetrahedral mesh around the embedded skeleton and applying the dynamic deformation method of Capell et al. [2002]. Combining retargetting with joint limits should eliminate some artifacts in the motion. A better retargetting scheme could be used to make animations more physically plausible and prevent global self-intersections. Finally, it would be nice to eliminate the assumption that the character must have a well-defined interior.

Beyond Pinocchio's current capabilities, an interesting problem is dealing with hand animation to give animated characters the ability to grasp objects, type, or speak sign language. The variety of types of hands makes this challenging (see, for example, Models 13, 5,14 , and 11 in Figure 9). Automatically rigging characters for facial animation is even more difficult, but a solution requiring a small amount of user assistance may succeed. Combined with a system for motion synthesis [Arikan et al. 2003], this would allow users to begin interacting with their creations.

\section{Acknowledgments}

We thank Yeuhi Abe and Eugene Hsu for help with motion capture. Thanks to Soonmin Bae, Inna Baran, Frédo Durand, Sylvain Paris, Ariel Shamir, Daniel Vlasic, and Robert Wang for their helpful feedback. Thanks to Emily Whiting for narrating the video. We 
thank Dragomir Anguelov for the human meshes. We would also like to thank Solidworks for the permission to use Cosmic Blobs models. This work was supported by a grant from Solidworks Corporation. The first author was also supported by an NSF Graduate Research Fellowship.

\section{References}

Anderson, D., Frankel, J. L., Marks, J., Agarwala, A., Beardsley, P., Hodgins, J., Leigh, D., Ryall, K., SulLIVAN, E., AND YedidiA, J. S. 2000. Tangible interaction + graphical interpretation: a new approach to $3 \mathrm{~d}$ modeling. In Proceedings of ACM SIGGRAPH 2000, Annual Conference Series, 393-402.

Arikan, O., Forsyth, D. A., And O’Brien, J. F. 2003. Motion synthesis from annotations. ACM Transactions on Graphics 22, 3 (July), 402-408.

BARAN, I., AND Popović, J., 2007. Penalty functions for automatic rigging and animation of $3 \mathrm{~d}$ characters. http://people.csail.mit.edu/ibaran/penalty.pdf.

BARAN, I., AND Popović, J., 2007. Pinocchio results video. http://people.csail.mit.edu/ibaran/pinocchio.avi.

Botsch, M., Bommes, D., And Kobbelt, L. 2005. Efficient linear system solvers for mesh processing. In IMA Conference on the Mathematics of Surfaces, 62-83.

Burges, C. 1998. A Tutorial on Support Vector Machines for Pattern Recognition. Data Mining and Knowledge Discovery 2, $2,121-167$.

Capell, S., Green, S., Curless, B., Duchamp, T., and Popović, Z. 2002. Interactive skeleton-driven dynamic deformation. ACM Transactions on Graphics 21, 3 (Aug.), 586-593.

CHOI, K.-J., AND Ko, H.-S. 2000. Online motion retargetting. Journal of Visualization and Computer Animation 11, 5 (Dec.), 223-235.

Frisken, S. F., Perry, R. N., Rockwood, A. P., ANd Jones, T. R. 2000. Adaptively sampled distance fields: A general representation of shape for computer graphics. In Proceedings of ACM SIGGRAPH 2000, Annual Conference Series, 249-254.

Gill, P. E., Murray, W., And Wright, M. H. 1989. Practical Optimization. Academic Press, London.

GLeicher, M. 2001. Comparing contraint-based motion editing methods. Graphical Models 63 (Aug.), 107-134.

Gold, S., And Rangarajan, A. 1996. A graduated assignment algorithm for graph matching. IEEE Transactions on Pattern Analysis and Machine Intelligence 18, 4, 377-388.

Igarashi, T., Matsuoka, S., And Tanaka, H. 1999. Teddy: A sketching interface for $3 \mathrm{~d}$ freeform design. In Proceedings of ACM SIGGRAPH 1999, Annual Conference Series, 409-416.

Igarashi, T., Moscovich, T., And Hughes, J. F. 2005. As-rigid-as-possible shape manipulation. ACM Transactions on Graphics 24, 3 (Aug.), 1134-1141.

Igarashi, T., Moscovich, T., And Hughes, J. F. 2005. Spatial keyframing for performance-driven animation. In Symposium on Computer Animation (SCA), 107-115.
JAROMCZYK, J. W., And Toussaint, G. T. 1992. Relative neighborhood graphs and their relatives. Proceedings of IEEE 80, 9 (Sept.), 1502-1517.

KatZ, S., AND TAL, A. 2003. Hierarchical mesh decomposition using fuzzy clustering and cuts. ACM Transactions on Graphics 22, 3 (Aug.), 954-961.

KAVAN, L., AND ŽÁRA, J. 2005. Spherical blend skinning: A realtime deformation of articulated models. In ACM SIGGRAPH Symposium on Interactive 3D Graphics and Games, 9-16.

KRY, P. G., James, D. L., AND PAI, D. K. 2002. EigenSkin: Real time large deformation character skinning in hardware. In Symposium on Computer Animation (SCA), 153-160.

Lipman, Y., Sorkine, O., Levin, D., And Cohen-Or, D. 2005. Linear rotation-invariant coordinates for meshes. $A C M$ Transactions on Graphics 24, 3 (Aug.), 479-487.

LIU, P.-C., Wu, F.-C., MA, W.-C., LIANG, R.-H., AND OUHYOUNG, M. 2003. Automatic animation skeleton using repulsive force field. In 11th Pacific Conference on Computer Graphics and Applications, 409-413.

Meyer, M., Desbrun, M., Schröder, P., And Barr, A. H. 2003. Discrete differential-geometry operators for triangulated 2-manifolds. In Visualization and Mathematics III. SpringerVerlag, Heidelberg, 35-57.

Moccozet, L., Dellas, F., Magnenat-Thalmann, N., BIasotti, S., Mortara, M., Falcidieno, B., Min, P., And VeltKAMP, R. 2004. Animatable human body model reconstruction from $3 \mathrm{~d}$ scan data using templates. In CapTech Workshop on Modelling and Motion Capture Techniques for Virtual Environments, 73-79.

NELDER, J., AND MEAD, R. 1965. A simplex method for function minimization. Computer Journal 7, 308-313.

Taskar, B., Guestrin, C., And Koller, D. 2003. Maxmargin markov networks. In Advances in Neural Information Processing Systems (NIPS 2003).

Teichmann, M., And Teller, S. 1998. Assisted articulation of closed polygonal models. In Computer Animation and Simulation '98, 87-102.

Thorne, M., Burke, D., And van de Panne, M. 2004. Motion doodles: an interface for sketching character motion. ACM Transactions on Graphics 23, 3 (Aug.), 424-431.

Toledo, S., 2003. TAUCS: A library of sparse linear solvers, version 2.2. http://www.tau.ac.il/ $\sim$ stoledo/taucs.

WADE, L. 2000. Automated generation of control skeletons for use in animation. $\mathrm{PhD}$ thesis, The Ohio State University.

Wang, R., Pulli, K., And Popović, J. 2007. Real-time enveloping with rotational regression. ACM Transactions on Graphics 26, 3. In press.

Yu, Y., Zhou, K., Xu, D., Shi, X., BaO, H., GuO, B., AND SHUM, H.-Y. 2004. Mesh editing with poisson-based gradient field manipulation. ACM Transactions on Graphics 23, 3 (Aug.), 644-651.

Zhou, K., Huang, J., Snyder, J., Liu, X., Bao, H., Guo, B., AND SHUM, H.-Y. 2005. Large mesh deformation using the volumetric graph laplacian. ACM Transactions on Graphics 24, 3 (Aug.), 496-503. 